\title{
Determinación de receptores de estrógeno beta en carcinomas mamarios caninos
}

\author{
Merlo, W.A.; Vargas, V.E.; Insfrán, R.M.; Benítez, J.S.; Rosciani, A.S.
}

Servicio de Diagnóstico Histopatológico y Citológico, Facultad de Ciencias Veterinarias, Universidad Nacional del Nordeste (UNNE), Sargento Cabral 2139, Corrientes (3400), Argentina. Tel: 379-4425753, interno 175.

E-mail:wmerlo@vet.unne.edu.ar.

\begin{abstract}
Resumen
Merlo, W.A.; Vargas, V.E.; Insfrán, R.M.; Benítez, J.S.; Rosciani, A.S.: Determinación de receptores de estrógeno beta en carcinomas mamarios caninos. Rev. vet. 24: 1, 10-13, 2013. El presente trabajo tuvo como objetivo determinar la presencia de receptores de estrógenos beta $(\mathrm{RE} \beta)$ en neoplasias mamarias malignas y relacionarla con otros parámetros de significación conocida. Se realizó la inmunomarcación por el sistema de biotinaestreptavidina-peroxidasa para RE $\beta$ en muestras obtenidas de 34 pacientes caninas con un seguimiento hasta dieciocho meses post cirugía. Se determinó el porcentaje de núcleos inmunomarcados según el tipo histológico (carcinoma simple: 51\%, carcinoma complejo: 62\%, carcinoma tumor mixto: 62\%) y el grado histológico de malignidad de las neoplasias (GHI: 60\% y GHII: 61\%); el estadio clínico (ECI: 69\%, ECII: 56\%, ECIII: 59\%, ECIV: 58\% y ECV: $58 \%$ ) y la sobrevida de las pacientes (vivas: 62\% y muertas: 60\%). Los resultados revelaron porcentajes de RE $\beta$ más elevados, sólo en algunos grupos de mejor pronóstico, en coincidencia con lo expresado por otros autores. Se demuestra que en la especie canina, los hallazgos comunicados no son uniformes con respecto al valor pronóstico de este marcador.
\end{abstract}

Palabras clave: canino, neoplasias mamarias, receptores de estrógeno beta, inmunohistoquímica.

\begin{abstract}
Merlo, W.A.; Vargas, V.E.; Insfrán, R.M.; Benítez, J.S.; Rosciani, A.S.: Determination of estrogen receptor beta in canine mammary carcinomas. Rev. vet. 24: 1, 10-13, 2013. This work aims to communicate the determination of estrogen receptor beta (ER $\beta)$ in mammary tumors of 34 canine patients whose evolution after eighteen months post surgery was known. Its presence was associated with clinical staging of patients, histological type, histological grading of the malignancy, and evolution. Immunostaining was performed by biotin-streptavidin-peroxidase system. Positive ER $\beta$ immunostaining was observed in nuclei of carcinoma cells, myoepithelial cells and chondrocites. It was determined the percentage of nuclei immunolabeled according to histological type (simple carcinoma: 51\%, complex carcinoma: 61\%, mixed carcinoma: 63\%), histologic grade of malignancy (GHI: $60 \%$ and GHII: 61\%), the clinical stage (ECI: 68\%, ECII: 56\%, ECIII: 59\%, ECIV: 58\% and ECV 58\%) and in accordance with survival at 18 months (live: $61 \%$ and dead: $60 \%$ ). These results show higher percentages in groups of better prognosis (although no significant differences were established) in coincidence with the opinions expressed by others, except in reference to the histological grade of malignancy. These conflicting data suggest that further analysis to assess ER $\beta$ prognostic potential in canine mammary tumors is needed.
\end{abstract}

Key words: dog, mammary neoplasms, estrogen receptor beta, immunohistochemistry.

\section{INTRODUCCIÓN}

La neoplasia mamaria canina es una patología hormona-dependiente con bastantes similitudes a su contraparte humana y la más frecuentemente observada en

Proyecto SGCYT-UNNE B020/09

Presentado Reunión Comunicaciones SGCYT 2011

Recibido: 21 diciembre 2012 / Aceptado: 4 abril 2013 la hembra canina no esterilizada ${ }^{12}$. Los estrógenos y la progesterona participan de manera relevante en el desarrollo tumoral, considerándose a estas hormonas como principales factores de riesgo de la enfermedad. Los análisis de los receptores de estrógenos y de progesterona son aceptados y usados como herramientas para la determinación del pronóstico y la predicción de la respuesta terapéutica hormonal en el cáncer mamario humano ${ }^{1}$. 
Con respecto a los receptores estrogénicos, han sido identificadas dos isoformas, alfa $(\mathrm{RE} \alpha)$ y beta $(\mathrm{RE} \beta)^{5}$. Las mujeres con cáncer mamario que evidencia $R E \alpha$, revelan mayores porcentajes de sobrevida y mejor respuesta al tratamiento hormonal ${ }^{7}$. El significado de la presencia de RE $\beta$ aún no está claro, aunque algunos investigadores le asignan un efecto protector antiproliferativo ${ }^{2,10}$. Los patrones de inmunorreactividad observados en los estudios de expresión de estos últimos, mostraron una marcada distribución nuclear, con reacciones muy débiles a nivel citoplasmático en las células de los carcinomas mamarios ${ }^{11}$.

En la especie canina, algunos autores consideran que la presencia de estos dos tipos de receptores estrogénicos tendría un comportamiento similar a lo referido acerca de los carcinomas de la mujer ${ }^{5,8}$. Se ha detectado la presencia de RE $\beta$ en células carcinomatosas, principalmente en tumores de tipo complejo y mixto $\mathrm{y}$ en menor medida en carcinomas simples ${ }^{5}$. Se han comunicado porcentajes de células tumorales positivas para RE $\beta$ de alrededor del $60 \%$, aunque dichas tasas varían irregularmente al tener en cuenta los estadios clínicos de las pacientes, observándose valores en ascenso en relación a etapas más avanzadas, con una disminución en el último estadio ${ }^{3}$.

Por otra parte, se han encontrado porcentajes elevados de RE $\beta$ en carcinomas inflamatorios ${ }^{4}$, los cuales manifiestan un comportamiento muy agresivo. Tales datos permiten inferir que, en la especie canina, los hallazgos comunicados con respecto a este marcador, no son uniformes en cuanto a su valor pronóstico.

En este trabajo se comunica la determinación de RE $\beta$ en tumores mamarios malignos de un grupo de pacientes caninas, con el propósito de relacionar su presencia con el tipo y grado histológico del tumor, así como el estadio clínico y la sobrevida de las pacientes.

\section{MATERIAL Y MÉTODOS}

Se estudiaron 34 hembras caninas con neoplasias mamarias que asistieron al Hospital de Clínicas de la Facultad de Ciencias Veterinarias de Corrientes (Argentina). Dichas pacientes, al momento de la primera consulta, fueron estadificadas clínicamente según una clasificación que incluye cinco estadios clínicos (ECI, ECII, ECIII, ECIV, ECV $)^{9}$. El seguimiento de las pacientes se prolongó hasta los 18 meses post-cirugía para determinar la sobrevida.

El estudio histopatológico de las neoplasias se realizó teniendo en cuenta la clasificación histológica de tumores mamarios del perro efectuada por la OMS ${ }^{6}$ y la graduación histológica de malignidad de dichos tumores ${ }^{7}$ sobre cortes coloreados con hematoxilina y eosina. Esta última determina tres grados de malignidad (GHI, GHII, GHIII) teniendo en cuenta la conservación de la arquitectura, el pleomorfismo nuclear y el número de mitosis.

Para la inmunomarcación se efectuaron cortes de 3 micrómetros y se utilizó el kit de biotina-streptavi- dina-peroxidasa (Supersensitive Immunodetection System, LP000-Ul, Biogenex, CA, USA). Se siguieron los lineamientos generales propuestos por el laboratorio proveedor de los anticuerpos. Luego de la inactivación de la peroxidasa endógena y del desenmascaramiento antigénico, los distintos cortes se incubaron con el anticuerpo primario RE $\beta$ (anticuerpo de conejo anti-humano: Anti-Estrogen Receptor beta-Biogenex Policlonal. Cat. $N^{o}$ PU385-UP).

Luego se realizó la incubación con el anticuerpo secundario marcado con biotina, a continuación se incorporó la peroxidasa-estreptavidina. El revelado se logró con el agregado de diaminobencidina (DAB) y finalmente se coloreó con hematoxilina como tinción de contraste. Todas las diluciones de anticuerpos primario, secundario, streptoavidina-peroxidasa y DAB se hicieron en buffer fosfato salino (PBS pH 7,4) conteniendo $5 \%$ de albúmina bovina. Se incluyeron controles positivos y negativos.

El recuento de células positivas para RE $\beta$ se efectuó promediando los núcleos inmunomarcados en 100 células tomadas al azar en tres campos de 400 aumentos para cada tumor. Estos hallazgos se agruparon de acuerdo con el tipo histológico, el grado histológico de malignidad, el estadio clínico y la sobrevida de las pacientes a 18 meses post cirugía.

Utilizando el programa InfoStat se efectuaron estadísticas descriptivas de los distintos parámetros clasificadores aquí considerados. Se obtuvo media aritmética, desvío estándar y coeficiente de variación para cada tipo histológico y para cada estadio clínico. Por medio del test de Student se investigó la aparición de diferencias significativas entre pacientes vivas y muertas (sobrevida) y entre los grados histológicos de malignidad.

\section{RESULTADOS}

Los hallazgos se consignan en la Tabla 1. Las comparaciones entre grupos no mostraron diferencias significativas.

Se observó la inmunomarcación positiva a RE $\beta$ de núcleos de células carcinomatosas (Figura 1), en cortes

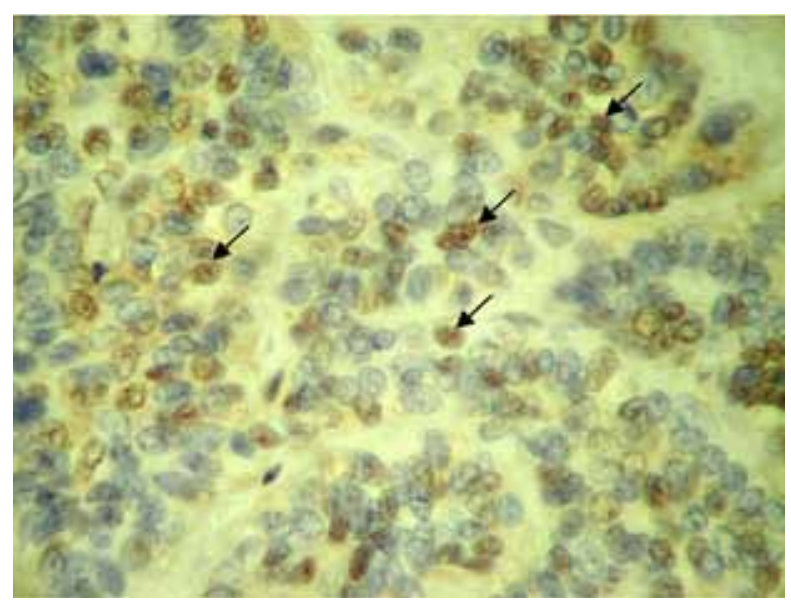

Figura 1. Carcinoma simple, GHI, núcleos de células carcinomatosas positivos a RE $\beta$ (flechas). IHQ, $400 \mathrm{x}$. 
Tabla 1. Presencia de receptores de estrógeno beta según el tipo histológico, grado histológico de malignidad, estadio clínico y evolución de las pacientes.

\begin{tabular}{lcccc}
\hline \multirow{2}{*}{ clasificación } & $\mathrm{N}^{\mathrm{o}}$ & \multicolumn{3}{c}{$\mathrm{RE} \beta$} \\
\cline { 5 - 6 } & casos & $\%$ & $\mathrm{DE}$ & $\mathrm{CV}$ \\
\hline Tipo histológico & & & & \\
carcinoma simple & 5 & 51 & 0,14 & 28,23 \\
carcinoma complejo & 12 & 62 & 0,13 & 21,33 \\
carcinoma en tumor mixto & 17 & 62 & 0,11 & 18,04 \\
Grado de malignidad & & & & \\
GHI & 28 & 60 & 0,13 & 21,37 \\
GHII & 6 & 61 & 0,13 & 21,04 \\
Estadio clínico & & & & \\
ECI & 7 & 69 & 0,09 & 13,34 \\
ECII & 4 & 56 & 0,06 & 10,50 \\
ECIII & 16 & 59 & 0,11 & 17,84 \\
ECIV & 5 & 58 & 0,18 & 31,96 \\
ECV & 2 & 58 & 0,33 & 56,08 \\
Evolución & & & & \\
vivas & 15 & 62 & 0,11 & 18,32 \\
muertas & 19 & 60 & 0,14 & 23,41 \\
\hline
\end{tabular}

RE $\beta$ : receptores de estrógeno beta; \%: promedio de núcleos positivos en 3 campos de 100 células; DE: desvío estándar; CV: coeficiente de variación; GHI, GHII: grado histológico de malignidad I y II; ECI, ECII, ECIII, ECIV, ECV: estadios clínicos I a V.

histológicos de los distintos tipos de carcinomas estudiados. Llamó la atención la reacción positiva de los núcleos de células mioepiteliales y condrocitos (Figuras 2 y 3$)$.

\section{DISCUSIÓN}

La isoforma de receptor estrogénico beta ha sido detectada en numerosas biopsias mamarias humanas, en líneas celulares de mama y en glándulas mamarias normales y tumorales caninas. En estas últimas, las neoplasias de naturaleza benigna presentan mayor expresión de este marcador, en tanto que, entre las malignas, los subtipos complejos y mixtos son los que evidencian porcentajes mayores de RE $\beta{ }^{5}$. Al mismo tiempo, la bibliografía clásica considera a estos últimos como neoplasias de mejor pronóstico que los carcinomas simples. Los hallazgos aquí obtenidos coinciden con tales afirmaciones. Se considera importante resaltar la positividad a RE $\beta$ mostrada por condrocitos de los carcinomas en tumor mixto y de diferentes células mioepiteliales de los carcinomas complejos y de los carcinomas en tumor mixto estudiados en el presente trabajo, circunstancias que no fueron halladas en la revisión bibliográfica efectuada.

Asímismo, los porcentajes de inmunomarcación de las pacientes que sobrevivieron y de las pacientes del ECI fueron más elevados, situación que sugeriría a este marcador como eventual indicador de buen pronóstico. Sin embargo, el porcentaje de núcleos reactivos en tumores de GHI fue más bajo en comparación

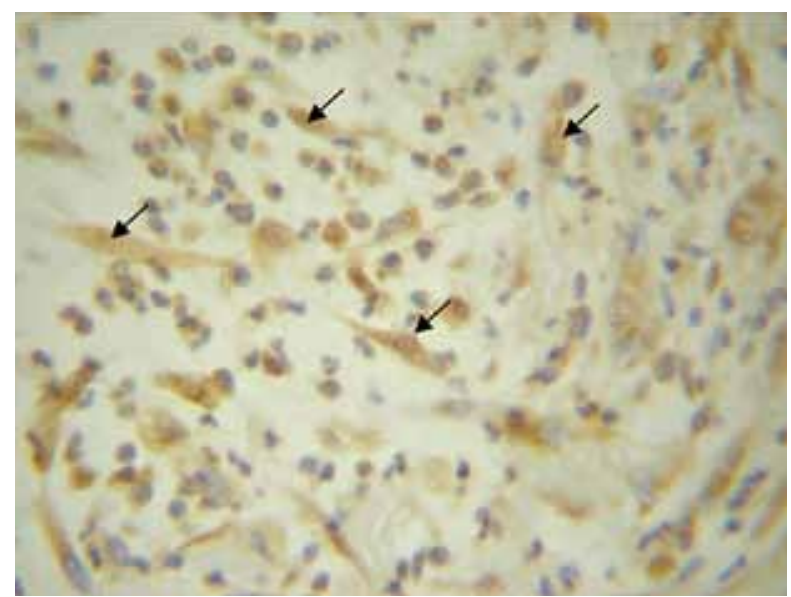

Figura 2. Carcinoma complejo, GHII, células mioepiteliales con núcleos positivos y citoplasmas con reacción leve (flechas). IHQ, 400x.

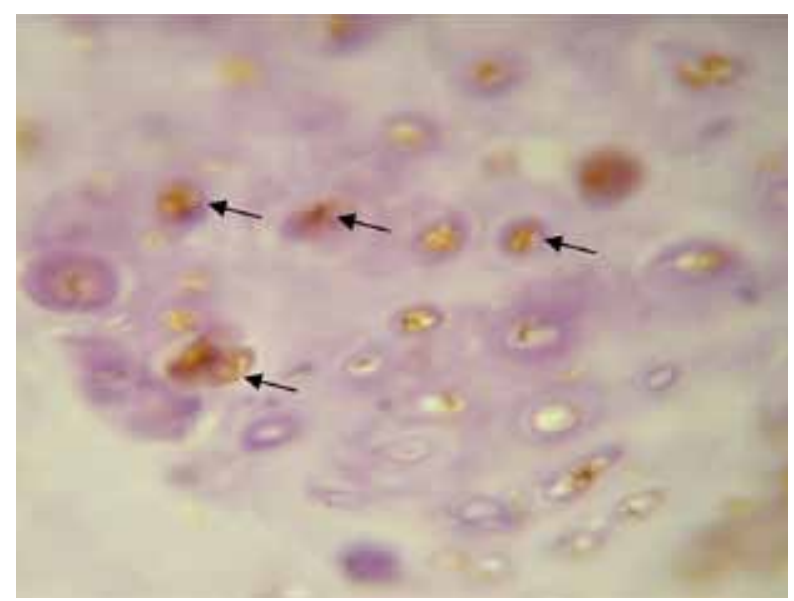

Figura 3. Carcinoma en tumor mixto, GHI, condrocitos con núcleos reactivos a RE $\beta$ (flechas). IHQ, $1000 \mathrm{x}$.

con el GHII. Debido a estas diferencias, se considera apropiado continuar el análisis de estos receptores para determinar su verdadero valor pronóstico en la especie canina y a la vez, estudiarlos en relación a otros marcadores como por ejemplo los RE $\alpha$ para poder determinar posibles grupos de tratamientos diferentes de acuerdo con su marcación, como en medicina humana.

\section{REFERENCIAS}

1. Allred DC, Harvey JM, Berardo M, Clark GM. 1998. Prognostic and predictive factors in breast cancer by immunohistochemical analysis. Mod Pathol 11: 155-168.

2. Gustafsson JA. 1999. Estrogen receptor: a new dimension in estrogen mechanism of action. J Endocr 163: 379-383.

3. Hermo G, Soldati R, Wargon V, Scursoni A, Lanari C, Gobello C. 2010. Inmunohistoquímica y expresión de receptores de estrógenos y progesterona en neoplasias mamarias malignas caninas en distintos estadios clínicos. Rev Científ FCV Univ Zuliá (Venezuela) 20: 245-253.

4. Illera JC, Pérez MD, Nieto A, Jiménez MA, Dunner S, Pen L. 2006. Steroids and receptors in canine mammary cancer. Steroids 71: 541-548. 
5. Martín J, Ordás J, Millán MY, Chacón M, De Lara F, Espinoza A, Reymundo C, Jover A. 2004. Immunohistochemical expression of estrogen receptor $\beta$ in normal and tumoral canine mammary glands. Vet Pathol 41: 269-272.

6. Misdorp W, Else RW, Hellmen E, Lipscomb TP. 1999. Histological classification of mammary tumors of the dog and the cat, Publ. World Health Org. (Washington DC), 2nd series, volume VII, p. 58.

7. Misdorp W. 2002. Tumors of the mammary gland. In: Tumors in domestic animals (Meuten DJ Ed), $4^{\text {th }}$ ed., Blackwell Publishing, Iowa, p. 575-606.

8. Nieto A, Peña L, Pérez Alenza MD, Sánchez MA, Flores JM, Castaño M. 2000. Immunohistologic detection of estrogen receptor alpha in canine mammary tumors: clinical and pathologic associations and prognostic significance. Vet Pathol 37: 239-247.

9. Owen L. 1980. TNM classification of tumors in domestic animals, Publ. World Health Organization, Geneva, $420 \mathrm{p}$.

10. Roger P, Sahla ME, Mäkela S, Gustafsson JA, Baldet P, Rochefort H. 2001. Decreased expression of estrogen receptor $\mathrm{b}$ protein in proliferative preinvasive mammary tumors. Cancer Res 61: 2537-2541.

11. Taylor AH, Al-Azzawi F. 2000. Immuno localisation of oestrogen receptor beta in human tissues. $J$ Mol Endocrinol 24:145-155.

12. Torres CG. 2005. Función del estradiol en el desarrollo de la neoplasia mamaria canina. Monogr Electron Patol Vet (Santiago, Chile) 2: 94-111.

\section{Revista Veterinaria obtuvo el máximo nivel de categorización del CAICYT-CONICET}

Tras el pertinente proceso de evaluación según criterios de calidad editorial, en setiembre de 2005 CAICYT-CONICET ha clasificado a nuestra publicación con Categoría 1 (nivel superior de excelencia), con lo cual pasa a integrar el Catálogo Latindex (folio 14022). La Dirección de Revista veterinaria agradece a quienes colaboraron para obtener tan importante distinción. Ver: http://www.latindex. unam.mx/busquedas/catalogotitulo.html 